This is a self-archived version of an original article. This version may differ from the original in pagination and typographic details.

Author(s): lancu, Edmond; Lappi, Tuomas; Triantafyllopoulos, Dionysios

Title: Small-x Physics in the Dipole Picture at NLO Accuracy

Year: 2020

Version: Accepted version (Final draft)

Copyright: (c) World Scientific, 2020

Rights: In Copyright

Rights url: http://rightsstatements.org/page/InC/1.0/?language=en

Please cite the original version:

Iancu, E., Lappi, T., \& Triantafyllopoulos, D. (2020). Small-x Physics in the Dipole Picture at NLO Accuracy. In A. Prokudin, Y. Hatta, Y. Kovchegov, \& C. Marquet (Eds.), Probing Nucleons and Nuclei in High Energy Collisions : Proceedings of INT Program INT-18-3 (pp. 274-294). World Scientific. https://doi.org/10.1142/9789811214950_0055 


\title{
Small- $x$ physics in the dipole picture at NLO accuracy
}

\author{
Edmond Iancu \\ Institut de Physique Thèorique, Université Paris-Saclay, CNRS, CEA, F-91191 Gif-sur-Yvette, \\ France \\ Tuomas Lappi \\ Department of Physics, P.O. Box 35, 40014 University of Jyväskylä, Finland and Helsinki \\ Institute of Physics, P.O. Box 64, 00014 University of Helsinki, Finland \\ Dionysios Triantafyllopoulos \\ European Centre for Theoretical Studies in Nuclear Physics and Related Areas (ECT*) and \\ Fondazione Bruno Kessler, Strada delle Tabarelle 286, I-38123 Villazzano (TN), Italy
}

\begin{abstract}
We review recent progress in NLO calculations for dilute-dense processes in the CGC picture. In particular, we focus here on recent steps in understanding high energy renormalization group evolution (BK/JIMWLK), the total DIS cross section at small $x$ and forward particle production in proton-nucleus collisions at next-to-leading order.

Keywords: Color Glass Condensate, Balitsky-Kovchegov equation, deep inelastic scattering
\end{abstract}

\section{Introduction}

The increasing accuracy of experimental data for small $x$ processes, both from the EIC and from ongoing LHC experiments, calls for a corresponding increase in the accuracy of theoretical calculations. This requires going to higher orders in perturbation theory also for processes where nonlinear gluon saturation phenomena are dominant. In order for the theory to have predictive power, it is crucial to have a consistent treatment of both inclusive and exclusive processes in DIS, and in forward rapidity proton-nucleus collisions and ultraperipheral collisions of heavy ions. In the high energy limit such a consistent, systematically perturbatively improveable, framework for describing the physics of gluon saturation is provided by the Color Glass Condensate (CGC) effective theory formulation. Here, the gluon fields of the target are described in terms of Wilson lines, path ordered exponentials in the strong color fields, which are measurable as the eikonal scattering amplitudes of a light projectile passing through the field.

Here we will review recent progress on NLO calculations in the CGC picture, concentrating on three different parts of the program towards a consistent framework for small- $x$ physics. We will first, in Sec. 2 discuss high energy (BK/JIMWLK) renormalization group evolution of the Wilson lines and their correlators, i.e. eikonal scattering amplitudes, with the energy scale of the process. We will then, in Sec. 3 move to calculations of the total DIS cross section in the dipole picture that natu- 
rally emerges in the CGC framework. Finally, in Sec. 4, we discuss single inclusive particle production in forward rapidity proton-nucleus collisions. Exclusive processes will be discussed in other contributions to this volume.

\section{High energy evolution}

Starting at next-to-leading order in perturbation theory, calculations of scattering processes at very high energy contain large logarithms of the center of mass energy $\sqrt{s}$ which can be resummed into a RG evolution. In terms of Wilson lines, which are appropriate for describing the scattering of small projectiles off a generic target, this evolution is given by the JIMWLK equation ${ }^{1-3}$. In most applications one only needs the scattering of a color dipole, whose evolution can be derived from the JIMWLK equation in a mean field approximation. The corresponding closed equation is known as the Balitsky-Kovchegov (BK) equation ${ }^{4,5}$.

\subsection{Double logarithms and instabilities}

The BK equation has now been derived up to NLO accuracy ${ }^{6-8}$. For the moment we shall take into account only the most dominant of the NLO terms, which also require a special treatment in order to lead to physically meaningful results. With $(\boldsymbol{x}, \boldsymbol{y})$ the transverse coordinates of the dipole, the equation of interest for the $S$-matrix $S_{\boldsymbol{x} \boldsymbol{y}}(Y)$ reads

$$
\frac{\partial S_{\boldsymbol{x} \boldsymbol{y}}}{\partial Y}=\frac{\bar{\alpha}_{s}}{2 \pi} \int \frac{\mathrm{d}^{2} \boldsymbol{z}(\boldsymbol{x}-\boldsymbol{y})^{2}}{(\boldsymbol{x}-\boldsymbol{z})^{2}(\boldsymbol{z}-\boldsymbol{y})^{2}}\left[1-\bar{\alpha}_{s} \ln \frac{(\boldsymbol{x}-\boldsymbol{z})^{2}}{(\boldsymbol{x}-\boldsymbol{y})^{2}} \ln \frac{(\boldsymbol{z}-\boldsymbol{y})^{2}}{(\boldsymbol{x}-\boldsymbol{y})^{2}}\right]\left(S_{\boldsymbol{x} \boldsymbol{z}} S_{\boldsymbol{z} \boldsymbol{y}}-S_{\boldsymbol{x} \boldsymbol{y}}\right),
$$

where $\bar{\alpha}_{s}=\alpha_{s} N_{\mathrm{c}} / \pi$ and with $N_{\mathrm{c}}$ the number of colors. We stress that the variable, for which the above equation has been derived, is the projectile rapidity $Y$. The dipole is a right moving object with large plus longitudinal momentum $q^{+}$and the target hadron a left moving object with minus longitudinal momentum, so that $s=2 q^{+} P^{-}$is the COM energy squared. If a typical parton in the target carries a fraction $x_{0}$ of $P^{-}$and if $Q_{0}$ is its typical transverse momentum, we define $p^{+}=Q_{0}^{2} / 2 x_{0} P^{-}$. Then the rapidity variable is defined as the boost invariant ratio

$$
Y=\ln \frac{q^{+}}{p^{+}}=\ln \frac{x_{0} s}{Q_{0}^{2}}=\ln \frac{x_{0}}{x} \frac{Q^{2}}{Q_{0}^{2}},
$$

where $Q^{2} \sim 1 / r^{2}$ with $r=|\boldsymbol{x}-\boldsymbol{y}|$ the dipole size and $x=Q^{2} / s$ as traditionally defined. In the kinematical limit of interest, $Y$ is assumed to be parametrically large. In general, the scattering amplitude $T_{\boldsymbol{x} \boldsymbol{y}}=1-S_{\boldsymbol{x} \boldsymbol{y}}$ is small when the target is dilute, while it approaches the unitarity limit $T_{\boldsymbol{x} \boldsymbol{y}}(Y)=1$ when the target is dense or "saturated". The two regimes are separated by a dynamically generated scale, the saturation momentum $Q_{s}(Y)$, which should be an increasing function of the rapidity $Y$. In other words, the scattering amplitude should increase with increasing $Y$ and fixed dipole size $r$, or with increasing $r$ and fixed rapidity $Y$. This 
is the physical expectation and this is indeed what happens when we keep only the LO term in Eq. (1) as one can verify both numerically and analytically.

The next natural step is to solve Eq. (1) including the "non-conformal" NLO term. In the regime where the daughter dipoles are large, i.e. for $|\boldsymbol{x}-\boldsymbol{z}| \simeq|\boldsymbol{z}-\boldsymbol{y}| \gg$ $r$, the double logarithm gets significantly large. For any reasonable value of the coupling $\bar{\alpha}_{s}$, the total contribution of the NLO term is larger in magnitude than the one of the LO term, and because of its negative sign it leads to a particularly awkward solution which exhibits an unphysical oscillating behavior. These features have been confirmed by analytical studies and numerical solutions ${ }^{9-11}$.

Such big collinear logarithms spoil the convergence of the perturbative expansion in $\bar{\alpha}_{s}$ and an all-orders resummation in the regime where large daughter dipoles are emitted, can be the only resolution to the problem. As is always the case in a Quantum Field Theory, large logarithmic contributions are associated with a certain physical mechanism. Here, they are generated by diagrams in light-cone perturbation theory in which the successive gluon (or dipoles at large- $N_{\mathrm{c}}$ ) emissions in the projectile are not only strongly ordered towards smaller (plus) longitudinal momenta and larger dipoles sizes, as clear from the above discussion, but are also ordered towards smaller lifetimes or equivalently larger light-cone energies ${ }^{11,12}$.

Simply by enforcing this time-ordering in the projectile wavefunction, we are led to a well-defined evolution which goes beyond a fixed order expansion in $\bar{\alpha}_{s}$. In fact there are two possibilities on how to proceed, and they are equivalent to the order of accuracy. In the one approach, the resummation of the double collinear logarithms is explicit in the evolution kernel and the ensuing equation reads ${ }^{11}$

$$
\frac{\partial S_{\boldsymbol{x} \boldsymbol{y}}}{\partial Y}=\frac{\bar{\alpha}_{s}}{2 \pi} \int \frac{\mathrm{d}^{2} \boldsymbol{z}(\boldsymbol{x}-\boldsymbol{y})^{2}}{(\boldsymbol{x}-\boldsymbol{z})^{2}(\boldsymbol{z}-\boldsymbol{y})^{2}} \mathcal{K}_{\mathrm{DLA}}\left(\rho_{\boldsymbol{x} \boldsymbol{y} \boldsymbol{z}}\right)\left(S_{\boldsymbol{x} \boldsymbol{z}} S_{\boldsymbol{z} \boldsymbol{y}}-S_{\boldsymbol{x} \boldsymbol{y}}\right),
$$

where the kernel is given by

$$
\mathcal{K}_{\mathrm{DLA}}(\rho) \equiv \frac{\mathrm{J}_{1}\left(2 \sqrt{\bar{\alpha}_{\mathrm{s}} \rho^{2}}\right)}{\sqrt{\bar{\alpha}_{s} \rho^{2}}} \quad \text { with } \quad \rho_{\boldsymbol{x} \boldsymbol{y} \boldsymbol{z}}^{2}=\ln \frac{(\boldsymbol{x}-\boldsymbol{z})^{2}}{(\boldsymbol{x}-\boldsymbol{y})^{2}} \ln \frac{(\boldsymbol{z}-\boldsymbol{y})^{2}}{(\boldsymbol{x}-\boldsymbol{y})^{2}}
$$

Eq. (3) remains a local equation in $Y$, like Eq. (1). When expanding to first nontrivial order in $\bar{\alpha}_{s}$ we recover the NLO double logarithm in Eq. (1). But the allorders kernel exhibits a completely different behavior at large distance: the Bessel function oscillates strongly when its argument gets large, and thus eliminates the contribution of large daughter dipoles which could violate time-ordering.

In the other approach the respective evolution equation reads ${ }^{12}$

$$
\begin{aligned}
\frac{\partial S_{\boldsymbol{x} \boldsymbol{y}}(Y)}{\partial Y}=\frac{\bar{\alpha}_{s}}{2 \pi} \int \frac{\mathrm{d}^{2} \boldsymbol{z}(\boldsymbol{x}-\boldsymbol{y})^{2}}{(\boldsymbol{x}-\boldsymbol{z})^{2}(\boldsymbol{z}-\boldsymbol{y})^{2}} \times \Theta\left(Y-\rho_{\min }\right) \\
\quad\left[S_{\boldsymbol{x} \boldsymbol{z}}\left(Y-\Delta_{\boldsymbol{x} \boldsymbol{z} ; r}\right) S_{\boldsymbol{z y}}\left(Y-\Delta_{\boldsymbol{z} \boldsymbol{y} ; r}\right)-S_{\boldsymbol{x} \boldsymbol{y}}(Y)\right],
\end{aligned}
$$

where the two quantities $\rho_{\min }$ and $\Delta_{\boldsymbol{x} z ; r}$ are given by

$$
\rho_{\min }=\ln \frac{1}{r_{\min }^{2} Q_{0}^{2}} \quad \text { and } \quad \Delta_{\boldsymbol{x} \boldsymbol{z} ; r}=\max \left\{0, \ln \frac{|\boldsymbol{x}-\boldsymbol{z}|^{2}}{r^{2}}\right\},
$$


and $r_{\min }$ is the size of the smallest of the three dipoles. Although we shall not show the details here, which require to write Eq. (5) in an integral form, the shifts in the arguments of the $S$-matrices are a direct consequence of the time-ordering constraint. Again there is a change w.r.t. the LO BK equation only regarding the contribution of the sufficiently large daughter dipoles. Contrary to Eq. (3), the above keeps the LO dipole kernel but is a non-local equation in $Y$. Equations (3) and (5) both reduce to the LO BK equation when expanded to LO in $\bar{\alpha}_{s}$, while they agree to all orders in $\bar{\alpha}_{s}$ so long as one is interested in the double logarithmic contributions arising from the emission of very large dipoles. The pure $\bar{\alpha}_{s}^{2}$ terms (i.e. terms of order $\mathcal{O}\left(\bar{\alpha}_{s}^{2}\right)$ not enhanced by large collinear logarithms) in the two equations are not the same, but they can be made to match each other and also match the respective terms of the full NLO BK equation.

Eqs. (3) and (5) have been solved as initial value problems ${ }^{13,14}$, using typical initial conditions like the MV model ${ }^{15,16}$. The solutions seem to be stable, but there are many issues in the results which are puzzling. The solution to the BK equation is always characterized by the speed $\lambda=\mathrm{d} \ln Q_{s}^{2} / \mathrm{d} Y$ of the evolution and the slope $\gamma=\mathrm{d} \ln T / \mathrm{d} \ln r^{2}$ in the perturbative side of the amplitude. The aforementioned equations lead to a somewhat low speed (such that after taking into account all the NLO corrections, it is difficult to cope with the phenomenology) and a significantly larger slope when compared to the LO dynamics. For fixed coupling evolution one finds that $\gamma$ is close to 1 or even larger than 1 , except for values of $\bar{\alpha}_{s}$ which are unnaturally small. Given that at LO the asymptotic value $\gamma \simeq 0.63$ is a hallmark of BFKL dynamics and saturation, one may wonder why this feature is lost when moving to NLO and beyond.

The first thing to realize, is that $Y$ is not the correct variable to use. It was merely introduced since in the presence of saturation it is much easier to calculate using the kinematics of the projectile which is a simple object. Instead, we should use the target rapidity $\eta$ defined as the logarithm of the ratio of the minus longitudinal momenta, namely

$$
\eta=\ln \frac{x_{0} P^{-}}{q^{-}}=\ln \frac{x_{0} s}{Q^{2}}=Y-\rho .
$$

We have defined the minus longitudinal momentum of the dipole according to $2 q^{-}=$ $Q^{2} / q^{+}$and the logarithmic variable $\rho=\ln 1 / r^{2} Q_{0}^{2}$. When doing DIS of a photon off the hadronic target, typically $Q^{2}$ is also the virtuality of the photon and thus $\eta$ is closely related to the logarithm of the Bjorken variable. Moreover, since we are mostly interested in a situation where $Q^{2} \gg Q_{0}^{2}$, the rapidities $Y$ and $\eta$ can be very different. We express the results in terms of the physical variable $\eta$ defining

$$
\bar{S}_{\boldsymbol{x y}}(\eta) \equiv S_{\boldsymbol{x y}}(Y=\eta+\rho),
$$

from which we extract the saturation momentum $Q_{s}^{2}(\eta)$. We find that indeed the evolution in terms of $\eta$ is faster, since the new speed reads $\bar{\lambda}=\lambda /(1-\lambda)$, while the shape of the amplitude is less steep since the new slope is given by $\bar{\gamma}=\gamma(1-\lambda)$. 
Still, there are two serious issues, which seem too difficult to deal with using the current procedure. First, we have not been very careful about the initial condition at $Y=0$ when solving Eqs. (3) or (5). In fact, these two equations are boundary value problems, more precisely a condition must be given at $Y=\rho$, but such a problem looks very hard to solve. A workaround, would be to write an initial condition at $Y=0$ which reproduces via evolution the desired amplitude, e.g. the MV model, at $Y=\rho$. This has been exactly done only at the level of linear evolution in the DLA $^{17}$, but it looks almost impossible to do it for the non-linear equation. Second, the shift $\Delta$ in Eq. (5), is not really uniquely defined as we have written in Eq. (6), rather it can be only specified within double-log accuracy. This arbitrariness in the choice of $\Delta$ leads to a small reasonable uncertainty in the results terms of $Y$. However, such a scheme dependence becomes very strong when expressing the results in terms of $\eta$ and leaves the whole approach without any predictive power ${ }^{17}$. For example, for certain schemes which at a first sight look fair, the speed $\bar{\lambda}$ turns out to be physically non-acceptable even for typical values of $\bar{\alpha}_{s}$.

\subsection{Evolution in the target rapidity}

The way to avoid the difficulties encountered at the end of Sect. 2.1 is rather simple. Instead of making the change of variables given in Eq. (8) on the solution, we shall do it directly on the evolution equation. Focusing on the non-local version in Eq. (5), it is an easy exercise to show that going from $(Y, \rho)$ to $(\eta, \rho)$ leads to ${ }^{17}$

$$
\begin{aligned}
& \frac{\partial \bar{S}_{\boldsymbol{x} \boldsymbol{y}}(\eta)}{\partial \eta}=\frac{\bar{\alpha}_{s}}{2 \pi} \int \frac{\mathrm{d}^{2} \boldsymbol{z}(\boldsymbol{x}-\boldsymbol{y})^{2}}{(\boldsymbol{x}-\boldsymbol{z})^{2}(\boldsymbol{z}-\boldsymbol{y})^{2}} \\
& \times \Theta\left(\eta-\delta_{\boldsymbol{x} \boldsymbol{y} \boldsymbol{z}}\right)\left[\bar{S}_{\boldsymbol{x} \boldsymbol{z}}\left(\eta-\delta_{\boldsymbol{x} \boldsymbol{z} ; r}\right) \bar{S}_{\boldsymbol{z} \boldsymbol{y}}\left(\eta-\delta_{\boldsymbol{z} \boldsymbol{y} ; r}\right)-\bar{S}_{\boldsymbol{x} \boldsymbol{y}}(\eta)\right],
\end{aligned}
$$

where the shifts are given by

$\delta_{\boldsymbol{x} \boldsymbol{z} ; r}=\ln \frac{|\boldsymbol{x}-\boldsymbol{z}|^{2}}{r^{2}}-\Delta_{\boldsymbol{x} \boldsymbol{z} ; r}=\max \left\{0, \ln \frac{r^{2}}{|\boldsymbol{x}-\boldsymbol{z}|^{2}}\right\}, \quad \delta_{\boldsymbol{x} \boldsymbol{y} \boldsymbol{z}}=\max \left\{\delta_{\boldsymbol{x} \boldsymbol{z} ; r}, \delta_{\boldsymbol{z} \boldsymbol{y} ; r}\right\}$.

We are still looking at the evolution from the projectile point of view, since the transverse sector involves the splitting of a dipole into two. The equation remains non-local where now the shift is effective only when one of the daughter dipoles is very small. When evolving in $\eta$ time ordering is trivially satisfied, while it is only due to the shift $\delta$ in Eq. (10) that $k^{+}$ordering is also guaranteed to hold. As before, the prescription for the shift is not unique beyond double-logarithmic accuracy. Finally, let us mention that we can truncate Eq. (9) to second order in $\bar{\alpha}_{s}$ by expanding to first order in the shift. Not surprisingly, the ensuing equation contains a large double logarithm when a small daughter dipole is formed. Although such an emission is atypical in the course of evolution, it can give rise to instabilities when $\eta$ gets large due to BFKL diffusion, and thus it is mandatory that one use Eq. (9) which effectively resums such logarithmic contributions to all orders in $\bar{\alpha}_{s}$.

The most essential feature of Eq. (9) is that, contrary to Eq. (5), it is an initial value problem. We can specify a physical condition $\bar{S}_{\boldsymbol{x} \boldsymbol{y}}(\eta=0)=\bar{S}_{\boldsymbol{x} \boldsymbol{y}}^{(0)}$ and proceed 
to solve the equation. The solution shows a weak dependence on the detailed form of the shift $\delta$, e.g. the uncertainty in the value of the speed $\bar{\lambda}$ is of order $\mathcal{O}\left(\bar{\alpha}_{s}^{2}\right)$, which is precisely what we should expect to the order of accuracy. Also the speed does not show any weird behavior, and the ratio $\bar{\lambda} / \bar{\alpha}_{s}$ is a monotonic function of $\bar{\alpha}_{s}$, namely it decreases when the coupling increases. Thus, we have a procedure which contains the proper physics in a controlled approximation.

\subsection{Beyond double logarithms}

So far we have focused on the higher order terms enhanced by double collinear logarithms, but there are other large corrections to be taken into account. The NLO BK equation also contains single transverse logarithms ${ }^{13}$, for which it is intuitively clear that they stand for DGLAP corrections on top of the small- $x$ evolution. That is, we can have a sequence of two emissions where only the first one is enhanced in the longitudinal sector, while both are strongly ordered in dipole sizes, either towards large daughter dipoles or towards dipoles of which one is much smaller. To a first approximation, such effects can be naturally resummed by inserting a power law suppression in the evolution kernel. More precisely, we introduce the factor

$$
\mathcal{K}_{A_{1}}=\left[\frac{(\boldsymbol{x}-\boldsymbol{y})^{2}}{\min \left\{(\boldsymbol{x}-\boldsymbol{z})^{2},(\boldsymbol{z}-\boldsymbol{y})^{2}\right\}}\right]^{ \pm A_{1}}
$$

with $A_{1}=11 / 12$ the familiar coefficient in the relevant DGLAP anomalous dimension. The sign in the exponent is such that $\mathcal{K}_{A_{1}}$ is never larger than one.

The last corrections that are mandatory to be included, are those related to the running coupling. We recall that on the r.h.s. of the NLO BK equation there is a term proportional to the first coefficient of the QCD $\beta$-function and which reads ${ }^{6,7}$

$$
\frac{\bar{b} \bar{\alpha}_{s}^{2}}{2 \pi} \int \frac{\mathrm{d}^{2} \boldsymbol{z}(\boldsymbol{x}-\boldsymbol{y})^{2}}{(\boldsymbol{x}-\boldsymbol{z})^{2}(\boldsymbol{z}-\boldsymbol{y})^{2}}\left[\ln (\boldsymbol{x}-\boldsymbol{y})^{2} \mu^{2}-\frac{(\boldsymbol{x}-\boldsymbol{z})^{2}-(\boldsymbol{y}-\boldsymbol{z})^{2}}{(\boldsymbol{x}-\boldsymbol{y})^{2}} \ln \frac{(\boldsymbol{x}-\boldsymbol{z})^{2}}{(\boldsymbol{y}-\boldsymbol{z})^{2}}\right] .
$$

Here $\bar{b}=\left(11 N_{\mathrm{c}}-2 N_{\mathrm{f}}\right) / 12 N_{\mathrm{c}}, N_{f}$ is the number of flavors and $\mu$ is a renormalization scale at which the coupling is evaluated. The logarithms can give large contributions and spoil the convergence of the perturbative expansion when either $r$ is very small or very large compared to $1 / \mu$ or when the gluon at $\boldsymbol{z}$ is close to any of the legs of the parent dipole. Thus $\mu$ must be chosen in such a way to guarantee that there are no large logarithms for any size of the three dipoles involved in the splitting process. Usually in QCD it is the hardest scale that determines the scale for the running coupling and one readily sees that this is true also here. The choice of $\mu$ is not unique and we shall list two different prescriptions which are equivalent to the order of accuracy. The first one is just the smallest dipole scheme defined by

$$
\bar{\alpha}_{\text {min }}=\bar{\alpha}_{s}\left(r_{\text {min }}\right) \quad \text { with } \quad r_{\min }=\min \{|\boldsymbol{x}-\boldsymbol{y}|,|\boldsymbol{x}-\boldsymbol{z}|,|\boldsymbol{z}-\boldsymbol{y}|\} .
$$

The second one is the BLM scheme, which in the current context means to choose 
$\mu$ so that the whole integrand in Eq. (12) vanishes. This leads to

$$
\bar{\alpha}_{\mathrm{BLM}}=\left[\frac{1}{\bar{\alpha}_{s}(|\boldsymbol{x}-\boldsymbol{y}|)}+\frac{(\boldsymbol{x}-\boldsymbol{z})^{2}-(\boldsymbol{z}-\boldsymbol{y})^{2}}{(\boldsymbol{x}-\boldsymbol{y})^{2}} \frac{\bar{\alpha}_{s}(|\boldsymbol{x}-\boldsymbol{z}|)-\bar{\alpha}_{s}(|\boldsymbol{z}-\boldsymbol{y}|)}{\bar{\alpha}_{s}(|\boldsymbol{x}-\boldsymbol{z}|) \bar{\alpha}_{s}(|\boldsymbol{z}-\boldsymbol{y}|)}\right]^{-1},
$$

and it is an elementary exercise to show that $\bar{\alpha}_{\text {BLM }}$ reduces to $\bar{\alpha}_{\text {min }}$ for all the configurations in which one of the three dipoles is much smaller than the other two.

Finally, we point out that there are other NLO corrections, which however are not enhanced by large logarithms in any kinematic regime. It suffices to say that the resummations which we reviewed here, can be matched to the full NLO BK equation. Thus, we have a stable formalism which includes the necessary resummations and is accurate to order $\mathcal{O}\left(\bar{\alpha}_{s}^{2}\right)$. In particular one must revise here the discussion given in Sect. 2.2, where the matching was done only at LO (cf. Eq. (9)) and this is why the uncertainty due to the choice of $\delta$ was of order $\mathcal{O}\left(\bar{\alpha}_{s}^{2}\right)$. When the matching is lifted to NLO accuracy, we can show that such an uncertainty due to the choice of a scheme is reduced to order $\mathcal{O}\left(\bar{\alpha}_{s}^{3}\right)$, as it should.

\section{Total DIS cross section}

The simplest scattering observable where the dipole $S$-matrix element $\mathcal{S}_{\boldsymbol{x} \boldsymbol{y}}$ appears is the total deep inelastic scattering cross section. Here the leading order picture is that the quark-antiquark dipole discussed in the context of the evolution equation is a quantum fluctuation of the virtual photon emitted by the lepton. Depending on the polarization state of the $\gamma^{*}$ one must consider separately transversally and longitudinally polarized photons. Experimentally the polarization states are separated using the kinematics of the scattered lepton and varying $\sqrt{s}$. Measuring both cross sections will be a central part of the physics program at the EIC.

In this "dipole factorization" one separates the process into an impact factor describing the fluctuation of the virtual photon into a partonic state, at leading order a dipole and at NLO also a $q \bar{q} g$ state, and the scattering amplitude of this state with the target, which at leading order is $1-S_{\boldsymbol{x} \boldsymbol{y}}$. The term "impact factor" is most often used for calculations in momentum space: for deep inelastic scattering at small- $x$ there is a momentum space calculation in Refs. 18,19. However, for the eikonal scattering picture in general, and for nonlinear high energy evolution in the CGC picture as discussed in Sec. 2, it is more convenient to work in mixed space, with transverse coordinates and longitudinal momentum. The calculation of the total DIS cross section in this formulation has been completed more recently in Refs. 20-22. This is the formulation we will discuss in more detail here.

The theoretical tool of choice for these calculations is light cone perturbation theory ${ }^{23-26}$. Here one calculates diagrammatically the perturbative expansion in terms of bare Fock states of the incoming particle (in this case $\gamma^{*}$ ) state. The coefficients of this expansion are known as the light cone wave functions. For the NLO DIS cross section the calculation is thus based on obtaining the virtual photon wavefunction to NLO accuracy. 


\subsection{Leading order}

In general, the total DIS cross section is obtained, via the optical theorem, from the forward limit of the elastic photon-target scattering amplitude

$$
\sigma^{\gamma^{*}}[A]=\left.\frac{2}{2 q^{+}(2 \pi) \delta\left(q^{+}-q^{+}\right)} \operatorname{Re}\left[{ }_{\mathrm{i}}\left\langle\gamma^{*}\left(\vec{q}^{\prime}, Q^{2}, \lambda^{\prime}\right)\left|1-\hat{S}_{E}\right| \gamma^{*}\left(\vec{q}, Q^{2}, \lambda\right)\right\rangle_{\mathrm{i}}\right]\right|_{\vec{q}^{\prime} \rightarrow \vec{q}}
$$

Here the photon states $\left|\gamma^{*}\left(\vec{q}, Q^{2}, \lambda\right)\right\rangle_{\mathrm{i}}$ are interacting theory states, that must be developed in a perturbation theory expansion in terms of the bare Fock states of the theory. The eikonal scattering operator $\hat{S}_{E}$ is diagonal in transverse position space, and in the CGC expressed in terms of Wilson lines in the target gluon field. At leading order, the only contributing photon Fock state is the quark-antiquark dipole, and the corresponding tree-level virtual photon wave functions are easily calculated. The resulting leading order expression

$$
\sigma_{T, L}^{\gamma^{*} p}\left(x, Q^{2}\right)=2 \sum_{f} \int \mathrm{d} z \mathrm{~d}^{2} \boldsymbol{b} \mathrm{d}^{2} \boldsymbol{r}\left|\Psi_{T, L}^{\gamma^{*} \rightarrow q \bar{q}}\right|^{2}(1-S(\boldsymbol{b}, \boldsymbol{r}, x)),
$$

has been widely used to describe HERA DIS data, with the dipole amplitude $S$ obtained from BK or JIMWLK evolution or from different more phenomenological parametrizations.

\subsection{Next to leading order}
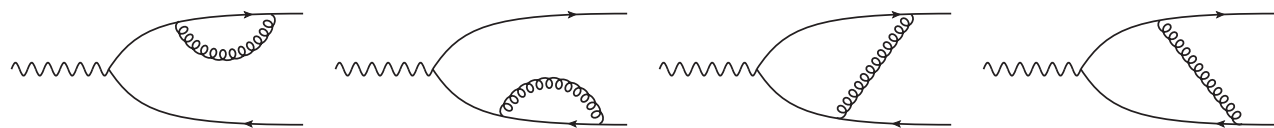

Fig. 1. Loop diagrams needed for the NLO $\gamma^{*} \rightarrow q \bar{q}$ wavefunction. In addition to these diagrams one must also include the corresponding "instantaneous interaction" contributions.
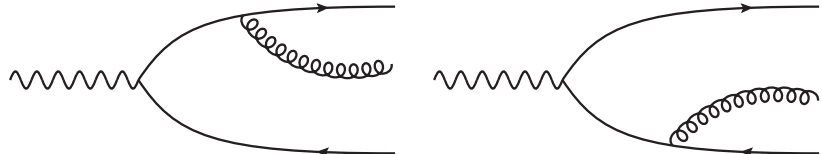

Fig. 2. Gluon emission diagrams needed for the $\gamma^{*} \rightarrow q \bar{q} g$ wavefunction. In addition to these diagrams one must also include the corresponding "instantaneous interaction" contributions.

At NLO accuracy one must develop the interacting photon state in Eq. (15) to include one loop corrections to the $\gamma^{*} \rightarrow q \bar{q}$ wavefunction, shown in Fig. 1, and the contribution of the $q \bar{q} g$ state, shown in Fig. 2. This calculation has been done in Refs. 20,21 in the conventional dimensional regularization (CDR) scheme 
and independently in the four-dimensional helicity (FDH) scheme in Ref. 22. The result can be written, using the notations of Ref. 22, as

$$
\sigma^{\gamma_{\mathrm{T}, \mathrm{L}}^{*}}[A]=\left.\sigma^{\gamma_{\mathrm{T}, \mathrm{L}}^{*}}\right|_{q \bar{q}}+\left.\sigma^{\gamma_{\mathrm{T}}^{*}}\right|_{q \bar{q} g},
$$

where the $q \bar{q}$ term is

$$
\begin{aligned}
\left.\sigma^{\gamma_{\mathrm{T}, \mathrm{L}}^{*}}\right|_{q \bar{q}}= & 2 \sum_{f} \int \mathrm{d} z \mathrm{~d}^{2} \boldsymbol{b} \mathrm{d}^{2} \boldsymbol{r}\left|\Psi_{T, L}^{\gamma^{*} \rightarrow q \bar{q}}\right|^{2} \\
& \times\left\{1+\left(\frac{\alpha_{s} C_{\mathrm{F}}}{\pi}\right)\left[\frac{1}{2} \log ^{2}\left(\frac{z}{1-z}\right)-\frac{\pi^{2}}{6}+\frac{5}{2}\right]\right\}(1-S(\boldsymbol{b}, \boldsymbol{r}, x))
\end{aligned}
$$

The $q \bar{q} g$ terms have slightly more complicated expressions ${ }^{20-22}$ that differ for the trasverse and longitudinal polarizations, and involve the $S$-matrix elements for the $q \bar{q} g$ Fock state scattering on the target

$$
S_{\boldsymbol{x} \boldsymbol{y} \boldsymbol{z}}=\frac{N_{\mathrm{c}}}{2 C_{\mathrm{F}}}\left[S_{\boldsymbol{x} \boldsymbol{z}} S_{\boldsymbol{z} \boldsymbol{y}}-\frac{1}{N_{\mathrm{c}}^{2}} S_{\boldsymbol{x} \boldsymbol{y}}\right] .
$$

where the nonlinear term is the same as in the BK equation as written in Eq. (3), and the second, $N_{\mathrm{c}}$-suppressed one, a part of the linear term.

Further details about the calculations leading to the NLO result can be found in the original references, so let us here make a few remarks on the slight differences between the two independent calculations. It is important to note that the split into the two terms (17) is not unique. Both the virtual (Fig. 1) and real (Fig. 2) separately contain divergences that, at least in light cone quantization, appear as logarithmic (transverse) UV ones. In coordinate space they correspond to the configurations where the gluon (at $\boldsymbol{z}$ ) is very close to its parent quark (at $\boldsymbol{x}$ ) or antiquark (at $\boldsymbol{y}$ ). In the calculation these divergences are regularized using transverse dimensional regularization, and cancel between the real and virtual terms in the end. This cancellation relies on the coincidence limit of the $q \bar{q}$ and $q \bar{q} g$ scattering amplitudes $S_{\boldsymbol{x} \boldsymbol{y} \boldsymbol{z}} \underset{\boldsymbol{z} \rightarrow \boldsymbol{x}}{\longrightarrow} S_{\boldsymbol{x} \boldsymbol{y}}$, which is always satisfied by their definitions in terms of Wilson line correlators. In practice the cancellation is effectuated by subtracting from the real part a divergent term proportional to $S_{\boldsymbol{x} \boldsymbol{y}}$. This subtraction term must have the correct form in the limits $\boldsymbol{z} \rightarrow \boldsymbol{x}$ and $\boldsymbol{z} \rightarrow \boldsymbol{y}$, but there is freedom in choosing its functional form for other values of $\boldsymbol{z}$. In addition to the different variant of the dimensional regularization scheme, the calculation in Refs. 20,21 on one hand and in Ref. 22 on the other hand, differ by the choice of this subtraction term. Thus the form in which the result is quoted is different, but the actual expressions equivalent.

The NLO impact factor contains a large logarithm of the energy (or of Bjorken $x$ in the context of DIS). In the DIS case this large logarithm resides in the $\left.\sigma^{\gamma_{\mathrm{T}}^{*}}\right|_{q \bar{q} g}$ term in Eq. (17), which contains an explicit integral over the longitudinal momentum fraction of the gluon in the diagrams of Figs. 1 and 2. When the gluon becomes very soft (i.e. the momentum fraction $z_{g} \rightarrow 0$ ), this integral yields a large 
logarithm of the lower cutoff, which kinematically is $\sim 1 / x_{\mathrm{Bj}}$. This contribution must be subtracted from the calculation of the cross section and absorbed into the $\mathrm{BK} / \mathrm{JIMWLK}$ evolution of the target in order to extract the finite genuinely NLO contribution. This factorization procedure is much less straightforward in the case of small $x$ than for collinear factorization. It is tied in with the issues of collinear logarithms or kinematical constraints in the NLO evolution discussed in 2. For DIS, there is a preliminary numerical implementation of the cross section formu$\mathrm{lae}^{27}$. This work shows that indeed the NLO corrections are sizeable (several tens of \%), but controllable. In particular, there is a large cancellation between the two different terms in Eq. (17), making the overall NLO correction smaller than the two separate terms individually. However, a more systematical comparison with experimental data has not yet been achieved, and it is not obvious if the factorization procedure here is the optimal one. The issues here are largely the same as in forward particle production, which has been discussed more in the recent literature. Thus we will not discuss this further here, but return to the issue in that context in Sec. 4.

\subsection{Massive quarks}

The calculation of the NLO DIS cross section discussed above was only performed with massless quarks. The total charm quark cross section is likely to be an important observable at the EIC. In particular, it is a more safely perturbative probe of weak coupling physics than the total cross section, being less sensitive to very large dipoles in the "aligned jet" (large $r$, small $z(1-z)$ ) configurations allowed even at large $Q^{2}$ for massless quarks ${ }^{28}$. Currently the work to extend the calculation described here to massive quarks is ongoing, and we shall briefly discuss the new issues involved.

In principle what one should calculate are precisely the same diagrams as for massless quarks. The presence of quark masses of course complicates somewhat the kinematics and the algebra in the loop integrals. More important, however, is that one must confront the known ${ }^{29-31}$ issues with quark mass renormalization in light cone perturbation theory.

To start, let us examine in more detail the structure of the elementary fermiongauge boson vertex of QED or QCD, for exaxmple for the emission of a gauge boson with momentum $k$ from a quark with momentum $p$, with the quark after the emission having 3-momentum $\vec{p}^{\prime}=\vec{p}-\vec{k}$ :

$$
\left[\bar{u}_{h^{\prime}}\left(p^{\prime}\right) d_{\lambda}^{*}(k) u_{h}(p)\right]
$$

Using the properties of the free spinors $u, \bar{u}, v, \bar{v}$ and polarization vectors $\varepsilon(k)$, and 3 momentum conservation, this vertex can be expressed in terms of three independent Lorentz structures: $\bar{u}_{h^{\prime}} \gamma^{+} u_{h} \delta^{i j} q^{i} \varepsilon_{\lambda}^{* j}, \bar{u}_{h^{\prime}} \gamma^{+}\left[\gamma^{i}, \gamma^{j}\right] u_{h} q^{i} \varepsilon_{\lambda}^{* j}$ and $\bar{u}_{h^{\prime}} \gamma^{+} \gamma^{j} u_{h} m_{q} \varepsilon_{\lambda}^{* j}$. The first two are light cone helicity conserving ones $\left(\sim \delta_{h, h^{\prime}}\right)$ that are present 
independently of the quark mass. The third one is a light cone helicity flip term $\sim \delta_{h,-h^{\prime}}$, and is explicitly proportional to the quark mass. Note also that the helicity flip vertex has one less power of the transverse momentum than the nonflip one, thus resulting in less UV divergent contributions.

The inclusion of the additional helicity flip structure introduces two new kinds mass-dependent UV divergent contributions. Firstly, in the first two diagrams of Fig. 1 one can have a flip vertex at both ends of the quark line. Since for massless quarks the transverse momentum integral in the loop is quadratically divergent, this yields a logarithmic UV divergence proportional to $m_{q}^{2}$. This divergence is absorbed into a renormalization of the quark mass squared appearing in the energy denominator of the leading order wave function. The corresponding counterterm is the "kinetic mass" counterterm, since it is associated with the kinetic property of the mass as a parameter in the dispersion relation relating the energy $p^{-}$to the momentum $p^{+}, \boldsymbol{p}$. Secondly, in the second two (vertex correction) diagrams of Fig. 1 one can take one out of the three vertices to have a flip, and get a logarithmically divergent contribution proportional to the quark mass $m_{q}$. This divergence, on the other hand, separately from the other one, is absorbed into a renormalization of the quark mass appearing in the helicity flip part of the leading order vertex. The associated counterterm is referred to as the "vertex mass" one, since it is related to the role of the quark mass as the coefficent of the helicity flip amplitude in an interaction with gauge bosons.

In a covariant formulation of the theory rotational invariance guarantees that both the kinetic and vertex masses remain the same at all orders in perturbation theory. However, in light cone quantization one chooses a specific coordinate axis as the longitudinal one. If the regularization method used in loop calculations is not rotationally invariant, the two counterterms can become different. This is indeed the case for the scheme of transverse dimensional regularization and a cutoff in the longitudinal $\left(p^{+}\right)$momentum that has been used in NLO calculations of DIS for mass. There are two possible ways to remedy this problem. One way is to introduce an additional renormalization condition to separately determine the two mass counterterms by enforcing rotational invariance order by order in perturbation theory. A convenient possibility or the case of DIS is to require that the decay amplitudes of transversally and longitudinally polarized timelike virtual photons are equal. The other option is to examine more closely the regularization procedure in the case of the problematic diagrams, which in this case are in fact the propagator correction ones. By carefully combining them with the corresponding instantaneous interaction diagrams before integration, and evaluating a specific subset of one-body phase space integrals in a rotationally invariant manner, one can restore rotational invariance without an additional renormalization condition. The full details of this procedure will be explained in more detail in a forthcoming publication. 


\section{Forward particle production in $p A$ collisions}

Particle production at forward rapidities and semi-hard transverse momenta in proton (or deuteron)-nucleus collisions at RHIC and the LHC is an important source of information about the small- $x$ part of the nuclear wavefunction, where gluon occupation numbers are high and non-linear effects like gluon saturation and multiple scattering are expected to be important. On the theory side, the cross-section for single-inclusive particle production has been computed ${ }^{32}$ up to next-to-leading order (NLO) in the framework of the so-called "hybrid factorization" 33 , but the result is problematic: the cross-section suddenly turns negative when increasing the transverse momentum of the produced hadron, while still in the semi-hard regime ${ }^{34}$. Various proposals to fix this difficulty, by modifying the scale for the rapidity subtraction, have only managed to push the problem to somewhat larger values of the transverse momentum ${ }^{35}$.

In a recent paper ${ }^{36}$, it has have argued that this negativity problem is an artefact of the approximations used within hybrid factorization in order to obtain a result which looks local in rapidity. On that occasion, it has also been proposed a more general factorization scheme, which is non-local in rapidity but yields a manifestly positive cross-section to NLO accuracy. Subsequently, this whole strategy has been extended to the calculation of the DIS structure functions at $\mathrm{NLO}^{27}$.

Another subtle issue refers to the use of a running coupling within the nonlocal factorization. Indeed, the cross-section is written in momentum space, but it involves the solution to the BK equation, which is most naturally solved in coordinate space; the mismatch between the respective prescriptions for the running of the coupling can lead into trouble ${ }^{37}$. A solution to this difficulty that was recently proposed $^{38}$ will be briefly reviewed here.

\subsection{Leading order formalism}

For simplicity, we focus here on the $q \rightarrow q$ channel and do not consider the fragmentation functions. To leading order (LO) in the "hybrid factorization", quark production at forward rapidities in $p A$ collisions proceeds as follows: a quark which is initially collinear with the incoming proton scatters off the dense gluon distribution in the nuclear target and thus acquires a transverse momentum $\boldsymbol{k}$. The LO quark multiplicity is computed as follows:

$$
\frac{\mathrm{d} N^{\mathrm{LO}}}{\mathrm{d}^{2} \boldsymbol{k} \mathrm{d} \eta}=\frac{x_{p} q\left(x_{p}\right)}{(2 \pi)^{2}} \mathcal{S}\left(\boldsymbol{k}, X_{g}\right), \quad \mathcal{S}\left(\boldsymbol{k}, X_{g}\right)=\int \mathrm{d}^{2} \boldsymbol{r} \mathrm{e}^{-\mathrm{i} \boldsymbol{k} \cdot \boldsymbol{r}} S\left(\boldsymbol{r}, X_{g}\right),
$$

where $\eta$ is the rapidity of the produced quark in the center-of-mass frame, $x_{p} q\left(x_{p}\right)$ is the quark distribution of the proton, and $x_{p}=\left(k_{\perp} \mathrm{e}^{\eta} / \sqrt{s}\right)$ and $X_{g}=\left(k_{\perp} \mathrm{e}^{-\eta} / \sqrt{s}\right)$ are the longitudinal momentum fractions carried by the partons participating in the collision - a quark from the proton and a gluon from the nucleus. The forward kinematics corresponds to $\eta$ positive and large, which implies $X_{g} \ll x_{p}<1$.

Furthermore, $\mathcal{S}\left(\boldsymbol{k}, X_{g}\right)$ is the relevant unintegrated gluon distribution (the 
"dipole TMD"), obtained as the Fourier transform of the $S$-matrix $S\left(\boldsymbol{r}, X_{g}\right)$ for the elastic scattering between a quark-antiquark color dipole with transverse size $\boldsymbol{r}$ and the nucleus. This quantity depends upon $X_{g}$ via the high energy evolution responsible for the rise in the gluon distributions with decreasing $X_{g}$. To the LO accuracy at hand, this evolution is described by (the LO version of the) the $\mathrm{BK}$ equation ${ }^{4,5}$, which resums to all orders the radiative corrections $\propto\left(\bar{\alpha}_{s} Y_{g}\right)^{n}$, with $Y \equiv \ln \left(1 / X_{g}\right)$. These corrections are associated with successive emissions of soft gluons, which are strongly ordered in longitudinal momenta and hence can be computed in the eikonal approximation.

The LO BK equation is boost invariant - it equivalently describes the highenergy evolution of the dipole projectile, or of the nuclear target. For what follows it is suggestive to vizualize this evolution in a Lorentz frame in which the "primary" gluon (the one which is closest in rapidity to the dipole) is emitted by the dipole, whereas all the other "soft" gluons belong to the nuclear wavefunction (see Fig. 3 for an illustration). Then the LO BK equation can be written in integral form,

$$
\begin{aligned}
S\left(\boldsymbol{x}, \boldsymbol{y} ; X_{g}\right)=S\left(\boldsymbol{x}, \boldsymbol{y} ; X_{0}\right) & +\frac{\bar{\alpha}_{s}}{2 \pi} \int_{X_{g} / X_{0}}^{1} \frac{\mathrm{d} x}{x} \int \frac{\mathrm{d}^{2} \boldsymbol{z}(\boldsymbol{x}-\boldsymbol{y})^{2}}{(\boldsymbol{x}-\boldsymbol{z})^{2}(\boldsymbol{z}-\boldsymbol{y})^{2}} \\
& \times[S(\boldsymbol{x}, \boldsymbol{z} ; X(x)) S(\boldsymbol{z}, \boldsymbol{y} ; X(x))-S(\boldsymbol{x}, \boldsymbol{y} ; X(x))],
\end{aligned}
$$

where $\boldsymbol{x}$ and $\boldsymbol{y}$ are the transverse coordinates of the quark and antiquark legs of the dipole (so, $\boldsymbol{r}=\boldsymbol{x}-\boldsymbol{y}$ ) and $\boldsymbol{z}$ is the transverse position of the primary gluon, which carries a fraction $x \ll 1$ of the longitudinal momentum of the incoming quark. Furthermore, $X_{0}$ is the value of $X$ at which one starts the high-energy evolution of the target, $S\left(\boldsymbol{x}, \boldsymbol{y} ; X_{0}\right)$ is the corresponding initial condition (say, as given by the McLerran-Venugopalan (MV) model ${ }^{15,16}$ ), and $X(x) \equiv X_{g} / x$ is the longitudinal momentum fraction of the gluons in the target which are probed by the scattering. Notice that $\ln (1 / X(x))=\ln \left(x / X_{g}\right)=Y_{g}-\ln (1 / x)$ is the rapidity separation between the primary gluon and the valence partons in the nucleus.

\subsection{The NLO impact factor}

At NLO, one needs to also include the "pure- $\alpha_{s}$ " corrections, i.e. the radiative corrections of $\mathcal{O}\left(\alpha_{s}\right)$ which are not enhanced by $Y_{g}$. These can be divided into two classes: (i) NLO corrections to the high-energy evolution, i.e. to the kernel (more generally, to the structure) of the BK equation, and (ii) NLO corrections to the "impact factor", i.e., to the "hard" matrix element which describes the quarknucleus scattering in the absence of any evolution, that is for $X_{g} \sim X_{0}$.

The LO impact factor describes the scattering between a bare quark collinear with the proton and the nucleus. At NLO, the wavefunction of the incoming quark may also contain a "primary" gluon with longitudinal fraction $x$ (cf. Fig. 3). For $x \ll 1$, this primary emission was already included in the LO evolution, as manifest in Eq. (22). The NLO correction to the impact factor is rather associated with a relatively hard primary emission, with $x \sim \mathcal{O}(1)$, which must be computed exactly 


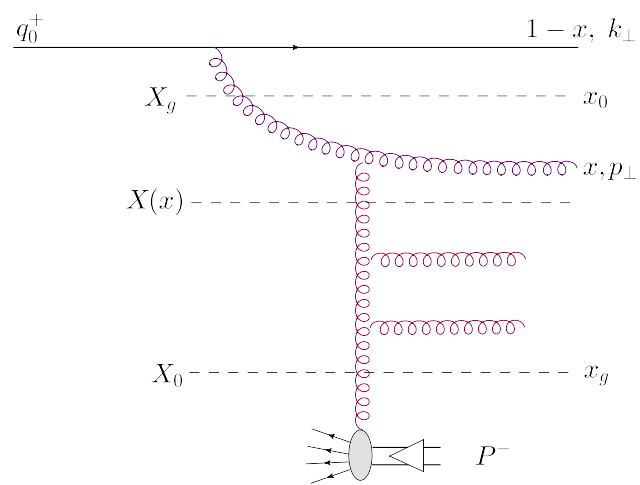

Fig. 3. A graph contributing to the amplitude for forward quark. When the longitudinal fraction $x$ of the "primary gluon" (the gluon directly emitted by the quark) is small, $x \ll 1$, this graph is a part of the high-energy evolution of the LO multiplicity. But for generic values $x \sim 1$ (non-eikonal emission), it contributes to the NLO impact factor.

(i.e. beyond the eikonal approximation). In practice though, it turns out that separating the LO evolution from the NLO correction to the impact factor is quite subtle. For this reason, we shall first present an "unsubtracted" expression for the NLO quark multiplicity ${ }^{36}$ in which these two effects are mixed with each other.

The LO multiplicity (21) receives NLO corrections proportional to the $N_{\mathrm{c}}$ and $C_{\mathrm{F}}$ color factors which have been computed in Refs. 32,39. To keep the discussion simple, we shall only consider the $N_{\mathrm{c}}$ terms which are ${ }^{40}$ the origin of the negativity problem observed in Ref. 34. Besides, we shall treat the dipole evolution to LO (the running coupling corrections will be later added). Finally, we shall focus on relatively hard momenta $k_{\perp} \gtrsim Q_{s}\left(X_{g}\right)$ for the produced quark, since this is the most interesting case for the phenomenology and also the regime where the negativity problem has been observed in the literature ${ }^{35}$. The sum of the LO and NLO contributions proportional to $N_{\mathrm{c}}$ can be written as ${ }^{36}$

$$
\frac{\mathrm{d} N^{\mathrm{LO}+N_{c}}}{\mathrm{~d}^{2} \boldsymbol{k} \mathrm{d} \eta}=\frac{x_{p} q\left(x_{p}\right)}{(2 \pi)^{2}} \mathcal{S}\left(\boldsymbol{k}, X_{0}\right)+\frac{\bar{\alpha}_{s}}{2 \pi} \int_{X_{g} / X_{0}}^{1} \frac{\mathrm{d} x}{x} \mathcal{K}(\boldsymbol{k}, x, X(x)),
$$

where the first term in the r.h.s. is the tree-level contribution (or equivalently the initial condition at $X_{g}=X_{0}$ ), whereas the second term encodes, in compact but rather formal notations, all the quantum corrections which are relevant to the accuracy of interest: the $N_{\mathrm{c}}$ piece of the NLO corrections to the impact factor and the LO BK evolution of the dipole $S$-matrix. The kernel $\mathcal{K}(\boldsymbol{k}, x, X(x))$ is built with vertices for the (generally, non-eikonal) emission of the primary gluon and with dipole $S$-matrices - evolved from $X_{0}$ down to $X(x)=X_{g} / x$ - describing the scattering between the nuclear target and the 2-parton projectile (the original quark plus the primary gluon). Explicit expressions can be found in Refs. 32,36,38,39.

We shall refer to the formula in Eq. (23) as "unsubtracted", since the NLO corrections are not explicitly separated from the LO result. This formula has been 

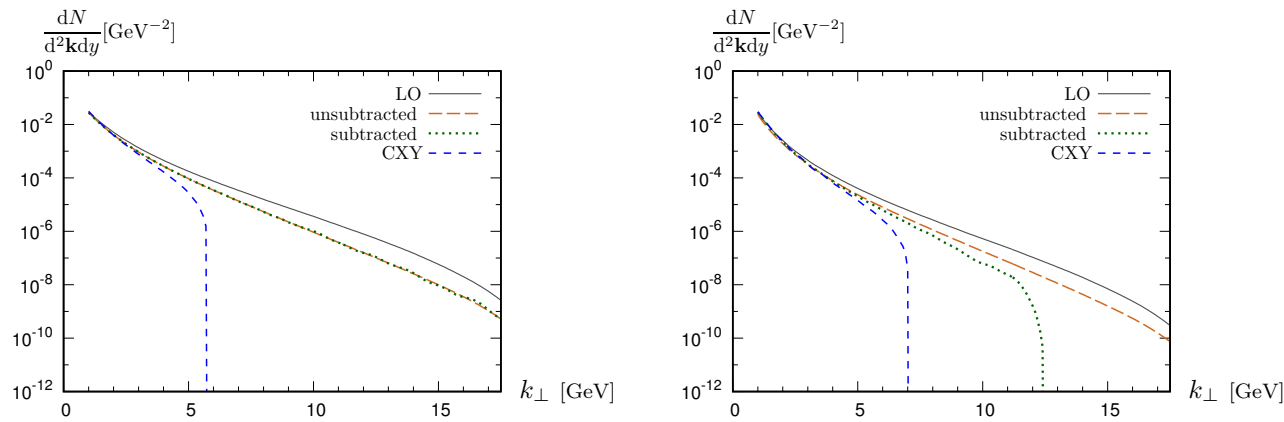

Fig. 4. Numerical results comparing the LO result, Eq. (21), with different formulations of the NLO factorization: "unsubtracted", cf. Eq. (23), "subtracted", cf. Eq. (25), and "CXY", cf. Eq. (26). Left: fixed coupling. Right: Running coupling with mixed RC prescriptions: transversespace prescription $\bar{\alpha}_{s}\left(k_{\perp}\right)$ for the primary gluon emission, but coordinate-space (Balitsky) prescription for the BK equation. These results are taken from Ref. ${ }^{37}$, to which we refer for more details.

numerically evaluated in Ref. 37, with the result shown in the left panel of Fig. 4 (the curve denoted as "unsubtracted"). This result is seen to be positive, as expected on physical grounds, and also smaller than the respective LO result - meaning that the NLO corrections are negative. In order to disentangle these corrections from the LO contribution and also make contact with the original calculations ${ }^{32,34,39}$, it is useful to observe that the LO result (21) can be recovered from Eq. (23) by taking the eikonal limit $x \rightarrow 0$ in the emission vertices (while keeping the $x$-dependence in the rapidity arguments $X(x)$ of the dipole $S$-matrices); that is,

$$
\frac{\mathrm{d} N^{\mathrm{LO}}}{\mathrm{d}^{2} \boldsymbol{k} \mathrm{d} \eta}=\frac{x_{p} q\left(x_{p}\right)}{(2 \pi)^{2}} \mathcal{S}\left(\boldsymbol{k}, X_{0}\right)+\frac{\bar{\alpha}_{s}}{2 \pi} \int_{X_{g} / X_{0}}^{1} \frac{\mathrm{d} x}{x} \mathcal{K}(\boldsymbol{k}, x=0, X(x)) .
$$

This is indeed consistent with equations (21) and (22), since the integral term above is the same as the Fourier transform of the respective term in Eq. (22) times $x_{p} q\left(x_{p}\right) /(2 \pi)^{2}$. By subtracting Eq. (24) from Eq. (23), one finds

$$
\frac{\mathrm{d} N^{\mathrm{LO}+N_{\mathrm{c}}}}{\mathrm{d}^{2} \boldsymbol{k} \mathrm{d} \eta}=\frac{\mathrm{d} N^{\mathrm{LO}}}{\mathrm{d}^{2} \boldsymbol{k} \mathrm{d} \eta}+\frac{\bar{\alpha}_{s}}{2 \pi} \int_{X_{g} / X_{0}}^{1} \frac{\mathrm{d} x}{x}[\mathcal{K}(\boldsymbol{k}, x, X(x))-\mathcal{K}(\boldsymbol{k}, x=0, X(x))] .
$$

The equivalence between Eqs. (23) and (25) is confirmed by the numerical study in Ref. 37: the "subtracted" curve in Fig. 4 (left), as obtained by numerically computing the r.h.s. of Eq. (25), perfectly matches the "unsubtracted" curve obtained from Eq. (23). But from the previous discussion, it should be clear that this equivalence relies in an essential way on the fact that the dipole $S$-matrix is an exact solution to the LO BK equation. Any approximation in solving this equation or in evaluating the NLO correction (the integral term) in the r.h.s. of Eq. (25) would lead to differences with potentially dramatic consequences. This observation is important in view of the fact that the "subtracted" formula (25) is not quite the same as the NLO prediction ${ }^{32,39}$ of the hybrid factorization (or the closely related $k_{T}$-factorization). 
The latter is local in rapidity, that is, the NLO correction to the impact factor is fully factorized from the high-energy evolution, which is evaluated at the scale $X_{g}$ (corresponding to the maximal rapidity separation between the projectile and the target). By contrast, the NLO correction in Eq. (25) is non-local in rapidity : it involves the dipole evolution at all the intermediate scales $X_{0}>X(x)>X_{g}$.

In order to arrive at the " $k_{T}$-factorized" formula presented in ${ }^{32,39}$, which is local in $X$, certain approximations need to be made. First, one observes that due to the subtraction in Eq. (25), the integral is dominated by large values $x \sim 1$. Hence, to the NLO accuracy of interest, it is justified to (i) replace the rapidity argument of the dipole $S$-matrices by its value at $x=1$, i.e. $X(x) \rightarrow X(1)=X_{g}$, and (ii) ignore the lower limit $X_{g} / X_{0} \ll 1$ in the integral over $x$. One thus obtains

$$
\left.\frac{\mathrm{d} N^{\mathrm{LO}+c}}{\mathrm{~d}^{2} \boldsymbol{k} \mathrm{d} \eta}\right|_{\mathrm{CXY}}=\frac{\mathrm{d} N^{\mathrm{LO}}}{\mathrm{d}^{2} \boldsymbol{k} \mathrm{d} \eta}+\int_{0}^{1} \frac{\mathrm{d} x}{x}\left[\mathcal{K}\left(\boldsymbol{k}, x, X_{g}\right)-\mathcal{K}\left(\boldsymbol{k}, x=0, X_{g}\right)\right],
$$

where all the $S$-matrices implicit in the r.h.s. are evaluated at the scale $X_{g}$.

Eq. (26) is not anymore equivalent to Eqs. (23) and (25) and, despite the seemingly reasonable approximations, it is rather pathological, as it rapidly becomes negative when increasing the transverse momentum of the produced quark. This is demonstrated by the curve "CXY" in the left panel of Fig. 4, obtained" 37 by numerically evaluating the r.h.s. of Eq. (26). The reason is that the replacement $X(x) \rightarrow X_{g}$ in the argument of the dipole $S$-matrix leads to an over-subtraction: the negative contribution proportional to $\mathcal{K}\left(\boldsymbol{k}, x=0, X_{g}\right)$ becomes too large in magnitude and overcompensates for the LO piece. Moreover, the replacement $X_{g} / X_{0} \rightarrow 0$ in the lower limit is not physically motivated, since it violates constraints imposed from the correct kinematics, and thus it introduces spurious contributions.

\subsection{Adding a running coupling}

So far, we have considered the academic case of a fixed coupling, but it is quite clear that, both for the consistency of the NLO calculation and for realistic applications to phenomenology, it is necessary to include the effects of the running of the coupling. Since the transverse momentum $k_{\perp}$ of the produced quark is the largest scale in the problem, it is intuitively clear that this is also the scale which controls the running of the coupling. It therefore looks reasonable to generalize the previous results by simply replacing $\bar{\alpha}_{s} \rightarrow \bar{\alpha}_{s}\left(k_{\perp}\right)$ in the primary emission vertices in equations (23) and (25), while simultaneously using rcBK (the LO BK equation with a running coupling) for the evolution of the dipole $S$-matrix. Yet, a moment of thinking reveals that such a procedure is not exempt of ambiguities, which in some cases may lead to serious problems. We now present several examples in that sense ${ }^{36-38}$.

In practice, it is preferable (for many good reasons ${ }^{37}$ ) to solve rcBK in the transverse coordinate space, meaning that the respective running coupling ( $\mathrm{RC})$ prescription must be formulated in coordinate space as well. Clearly, the scale 
dependence of the RC does not "commute" with the Fourier transform (FT), e.g.

$$
\bar{\alpha}_{s}\left(k_{\perp}\right) \mathcal{K}(\boldsymbol{k}, x, X(x)) \neq \int \mathrm{d}^{2} \boldsymbol{r} \mathrm{e}^{-\mathrm{i} \boldsymbol{k} \cdot \boldsymbol{r}} \bar{\alpha}_{s}\left(r_{\perp}\right) K(\boldsymbol{r}, x, X(x)),
$$

where $K(\boldsymbol{r}, x, X(x))$ is the $\mathrm{FT}$ of $\mathcal{K}(\boldsymbol{k}, x, X(x))$. One may interpret this mismatch as merely a part of our scheme dependence, but in some cases it may have dramatic consequences.

First, it spoils the equivalence between the "unsubtracted" and "subtracted" expressions for the NLO multiplicity. Recall indeed that in going from Eq. (23) to Eq. (25), we have used the fact that the integral term in Eq. (24) coincides with the Fourier transform of the r.h.s. of the LO BK equation. Clearly, this property is spoilt after replacing $\bar{\alpha}_{s} \rightarrow \bar{\alpha}_{s}\left(k_{\perp}\right)$ in Eq. (24), while at the same time using the coordinate-space version of the rcBK equation. Due to the fine-tuning inherent in the derivation of the "subtracted" expression, any such a mismatch could lead to a resurgence of the negativity problem. This is indeed observed by the numerical study in ${ }^{37}$ (see the right plot of Fig. 4): whereas the "unsubtracted" result remains positive and shows a similar trend as at fixed coupling, the "subtracted" one eventually turns negative, albeit at some larger value for $k_{\perp}$ than for the " $k_{T^{-}}$ factorized" expression (26) (now extended to a RC).

In order to cope with such issues, an alternative numerical implementation in which the calculation is fully performed in coordinate space was suggested ${ }^{37}$. In particular, the integral term in Eqs. (23) or (25) is constructed as in the r.h.s. of Eq. (27), that is, as the FT of a quantity originally computed in coordinate space. To get more insight on the role of the $\mathrm{RC}$ in this context, it is instructive to consider the eikonal limit $x \rightarrow 0$, in which Eq. (23) reduces to the LO result in Eq. (24). In coordinate space and with a RC, the integrand there should be understood as

$$
" \bar{\alpha}_{s}\left(r_{\perp}\right) K(\boldsymbol{r}, x=0)^{\prime \prime}=\frac{x_{p} q\left(x_{p}\right)}{(2 \pi)^{2}} \int \frac{\mathrm{d}^{2} \boldsymbol{x}}{2 \pi} \frac{\bar{\alpha}_{s}(\boldsymbol{r}) \boldsymbol{r}^{2}}{\boldsymbol{x}^{2}(\boldsymbol{x}-\boldsymbol{r})^{2}}[S(\boldsymbol{x}) S(\boldsymbol{r}-\boldsymbol{x})-S(\boldsymbol{r})],
$$

where $\bar{\alpha}_{s}(\boldsymbol{r})$ within the integrand generically refers to any coordinate-space RC prescription which also depends upon the size $\boldsymbol{r}$ of the parent dipole; e.g., the smallest dipole prescription $\bar{\alpha}_{s}\left(r_{\min }\right)$, with $r_{\min } \equiv \min \{|\boldsymbol{r}|,|\boldsymbol{x}|,|\boldsymbol{r}-\boldsymbol{x}|\}$. The generalization of Eq. (28) to generic (non-eikonal) values of $x$ can be found in Refs. 37,38.

The final results for the NLO quark multiplicity obtained via this procedure ${ }^{37}$ turned out to be extremely peculiar and physically unacceptable: not only they are dramatically different from the results obtained with the momentum-space prescription $\bar{\alpha}_{s}\left(k_{\perp}\right)$, but they also show an unphysical trend: the NLO corrections are very large and positive, and rapidly increase with $k_{\perp}$ (see the comparison between the curves "rcBK $\left(r_{\min }\right)$ " and " $\bar{\alpha}_{s}\left(k_{\perp}\right)$ " in the left plot in Fig. 5$)$.

The origin of this problem and also a solution to it have been identified ${ }^{38}$. Once again, this is related to the non-commutativity of the $\mathrm{RC}$ with the FT. We have no place here to explain the precise technical problem in the context of the NLO multiplicity, but we can illustrate it with a simpler example ${ }^{38}$ : this is Eq. (27) with 

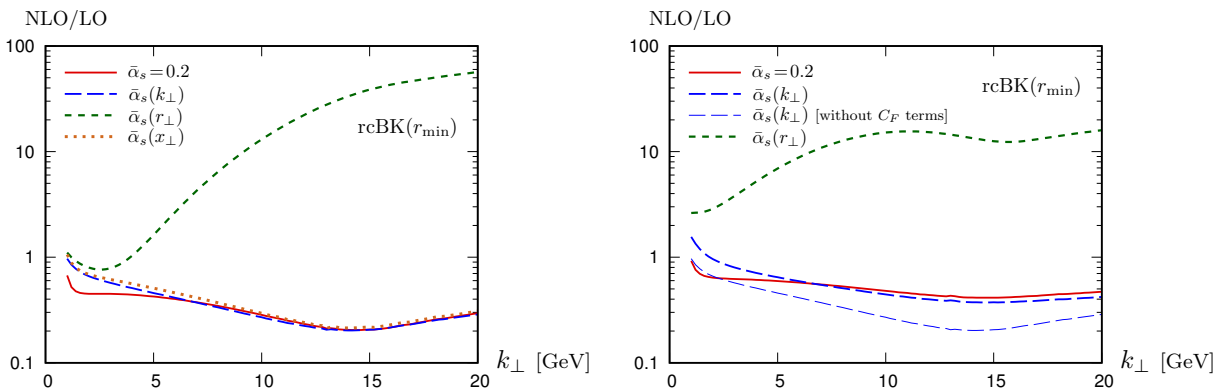

Fig. 5. Left: Ratio of the NLO multiplicity (including only the $N_{\mathrm{c}}$ terms) and the LO one for different prescriptions of the running coupling. Right: Ratio of the total NLO quark multiplicity (including both the $N_{\mathrm{c}}$ and $C_{\mathrm{F}}$ terms) and the LO one for three running coupling prescriptions. For comparison, we also show the results for $\bar{\alpha}_{s}\left(k_{\perp}\right)$ when including only the $N_{\mathrm{c}}$ terms (same as the curve " $\bar{\alpha}_{s}\left(k_{\perp}\right)$ " in the left panel). For both figures $\sqrt{s}=500 \mathrm{GeV}, \eta=3.2$ and the evolution of the color dipoles is obtained by solving the Balitsky-Kovchegov equation with the smallest dipole prescription using an $\mathrm{MV}^{16}$ initial condition at $X_{0}=0.01$.

the kernel $K$ replaced by the dipole $S$-matrix, evaluated at tree-level and in the single scattering approximation: $S=1-T$ with $T=r_{\perp}^{2} Q_{s}^{2} \ln \frac{1}{r_{\perp}^{2} \Lambda^{2}}$. The FT of $S$ (i.e. the analog of the l.h.s. of Eq. (27)) is controlled by the logarithmic singularity of $T$ as $r_{\perp} \rightarrow 0$, which yields

$$
\bar{\alpha}_{s}\left(k_{\perp}\right) \int \mathrm{d}^{2} \boldsymbol{r} \mathrm{e}^{-\mathrm{i} \boldsymbol{k} \cdot \boldsymbol{r}}\left(-r_{\perp}^{2} Q_{s}^{2} \ln \frac{1}{r_{\perp}^{2} \Lambda^{2}}\right)=\frac{16 \pi \bar{\alpha}_{s}\left(k_{\perp}\right) Q_{s}^{2}}{k_{\perp}^{4}} .
$$

This is the expected result for the high- $k_{\perp}$ tail of the quark multiplicity within the MV model. On the other hand, when inserting the $\mathrm{RC} \bar{\alpha}_{s}(\boldsymbol{r})$ inside the integrand, the mathematics goes very differently: the $\mathrm{RC}$ itself has a logarithmic singularity as $r_{\perp} \rightarrow 0$, due to the asymptotic freedom, which now dominates the FT:

$$
\int \mathrm{d}^{2} \boldsymbol{r} \mathrm{e}^{-\mathrm{i} \boldsymbol{k} \cdot \boldsymbol{r}} \bar{\alpha}_{s}\left(r_{\perp}\right) S(\boldsymbol{r}) \simeq \int \mathrm{d}^{2} \boldsymbol{r} \mathrm{e}^{-\mathrm{i} \boldsymbol{k} \cdot \boldsymbol{r}} \bar{\alpha}_{s}\left(r_{\perp}\right) \simeq-\frac{4 \pi}{\bar{b}\left[\ln \left(k_{\perp}^{2} / \Lambda^{2}\right)\right]^{2}} \frac{1}{k_{\perp}^{2}} .
$$

(We have used $\bar{\alpha}_{s}\left(r_{\perp}\right)=\left[\bar{b} \ln \frac{1}{r_{\perp}^{2} \Lambda^{2}}\right]^{-1}$.) Both the sign and the power law tail in Eq. (30) are different from the correct ones in Eq. (29). This is very similar with the results for the coordinate-space calculation " $r c B K\left(r_{\min }\right)$ " in Fig. 5.

This example suggests that the difficulty encountered with the coordinate-space calculation in Ref. 37 is due to the fact that the argument of the RC depends upon the parent dipole size $\boldsymbol{r}$ (the coordinate involved in the FT). Accordingly, the solution to this problem as suggested in Ref. 38 consists in using a different RC prescription, which is independent of $\boldsymbol{r}$ and hence commutes with the FT: in the notations of Eq. (28), this is the daughter dipole prescription $\bar{\alpha}_{s}\left(x_{\perp}\right)$. With this prescription, the coordinate-space calculation becomes remarkably close to that using the momentum-space prescription $\bar{\alpha}_{s}\left(k_{\perp}\right)$ (see the left plot in Fig. 5).

This being said, the daughter dipole prescription is not ideal either: First, it is so finely-tuned that one cannot study the scheme-dependence of the calculation. 
Second, it cannot be extended to the NLO corrections proportional to the quark Casimir $C_{\mathrm{F}}$. We thus consider that the most physical choice in the general case is the momentum space prescription $\bar{\alpha}_{s}\left(k_{\perp}\right)$. In Fig. 5 (right) we show the results we obtain when including both the $N_{\mathrm{c}}$ and $C_{\mathrm{F}}$ NLO corrections with fixed, momentumspace and coordinate-space RC. For comparison we also show the results obtained with the momentum space prescription including only the $N_{\mathrm{c}}$ NLO terms. This allows us to see that the inclusion of the $C_{\mathrm{F}}$ terms has a sizable effect and, being opposite in sign compared to the $N_{\mathrm{c}}$ terms, they reduce the size of the NLO corrections to the cross-section. This cancellation is similar to the one for the DIS cross section discussed in Sec. 3 between the $q \bar{q}$-term (with color factor $C_{\mathrm{F}}$ ) and the $q \bar{q} g$-term that has the color factor $N_{\mathrm{c}}$ of the BK equation.

Acknowledgments The work of E.I. was supported in part by the Agence Nationale de la Recherche project ANR-16-CE31-0019-01. T. L. has been supported by the Academy of Finland, project 321840, and by the European Research Council, grant ERC-2015-CoG-681707. The content of this article does not reflect the official opinion of the European Union and responsibility for the information and views expressed therein lies entirely with the authors.

\section{References}

1. J. Jalilian-Marian, A. Kovner, A. Leonidov and H. Weigert, The Wilson renormalization group for low x physics: Towards the high density regime, Phys. Rev. D59, p. 014014 (1998).

2. E. Iancu, A. Leonidov and L. D. McLerran, Nonlinear gluon evolution in the color glass condensate. 1., Nucl. Phys. A692, 583 (2001).

3. E. Ferreiro, E. Iancu, A. Leonidov and L. McLerran, Nonlinear gluon evolution in the color glass condensate. 2., Nucl. Phys. A703, 489 (2002).

4. I. Balitsky, Operator expansion for high-energy scattering, Nucl. Phys. B463, 99 (1996).

5. Y. V. Kovchegov, Small-x F2 structure function of a nucleus including multiple pomeron exchanges, Phys. Rev. D60, p. 034008 (1999).

6. I. Balitsky, Quark contribution to the small-x evolution of color dipole, Phys. Rev. D75, p. 014001 (2007).

7. Y. V. Kovchegov and H. Weigert, Triumvirate of Running Couplings in Small-x Evolution, Nucl. Phys. A784, 188 (2007).

8. I. Balitsky and G. A. Chirilli, Next-to-leading order evolution of color dipoles, Phys. Rev. D77, p. 014019 (2008).

9. E. Avsar, A. M. Stasto, D. N. Triantafyllopoulos and D. Zaslavsky, Next-to-leading and resummed BFKL evolution with saturation boundary, JHEP 10, p. 138 (2011).

10. T. Lappi and H. Mäntysaari, Direct numerical solution of the coordinate space Balitsky-Kovchegov equation at next to leading order, Phys. Rev. D91, p. 074016 (2015).

11. E. Iancu, J. D. Madrigal, A. H. Mueller, G. Soyez and D. N. Triantafyllopoulos, Resumming double logarithms in the QCD evolution of color dipoles, Phys. Lett. B744, 293 (2015).

12. G. Beuf, Improving the kinematics for low- $x$ QCD evolution equations in coordinate space, Phys. Rev. D89, p. 074039 (2014). 
13. E. Iancu, J. D. Madrigal, A. H. Mueller, G. Soyez and D. N. Triantafyllopoulos, Collinearly-improved BK evolution meets the HERA data, Phys. Lett. B750, 643 (2015).

14. T. Lappi and H. Mäntysaari, Next-to-leading order Balitsky-Kovchegov equation with resummation, Phys. Rev. D93, p. 094004 (2016).

15. L. D. McLerran and R. Venugopalan, Gluon distribution functions for very large nuclei at small transverse momentum, Phys. Rev. D49, 3352 (1994).

16. L. D. McLerran and R. Venugopalan, Computing quark and gluon distribution functions for very large nuclei, Phys. Rev. D49, 2233 (1994).

17. B. Ducloué, E. Iancu, A. H. Mueller, G. Soyez and D. N. Triantafyllopoulos, Non-linear evolution in QCD at high-energy beyond leading order, JHEP 04, p. 081 (2019).

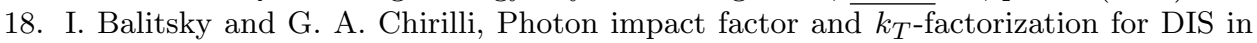
the next-to-leading order, Phys. Rev. D87, p. 014013 (2013).

19. I. Balitsky and G. A. Chirilli, Photon impact factor in the next-to-leading order, Phys. Rev. D83, p. 031502 (2011).

20. G. Beuf, Dipole factorization for DIS at NLO: Loop correction to the $\gamma_{T, L}^{*} \rightarrow q \bar{q}$ light-front wave functions, Phys. Rev. D94, p. 054016 (2016).

21. G. Beuf, Dipole factorization for DIS at NLO: Combining the $q \bar{q}$ and $q \bar{q} g$ contributions, Phys. Rev. D96, p. 074033 (2017)

22. H. Hänninen, T. Lappi and R. Paatelainen, One-loop corrections to light cone wave functions: the dipole picture DIS cross section, Annals Phys. 393, 358 (2018).

23. J. B. Kogut and D. E. Soper, Quantum Electrodynamics in the Infinite Momentum Frame, Phys. Rev. D1, 2901 (1970).

24. J. D. Bjorken, J. B. Kogut and D. E. Soper, Quantum Electrodynamics at Infinite Momentum: Scattering from an External Field, Phys. Rev. D3, p. 1382 (1971).

25. G. P. Lepage and S. J. Brodsky, Exclusive Processes in Perturbative Quantum Chromodynamics, Phys. Rev. D22, p. 2157 (1980).

26. S. J. Brodsky, H.-C. Pauli and S. S. Pinsky, Quantum chromodynamics and other field theories on the light cone, Phys. Rept. 301, 299 (1998).

27. B. Ducloué, H. Hänninen, T. Lappi and Y. Zhu, Deep inelastic scattering in the dipole picture at next-to-leading order, Phys. Rev. D96, p. 094017 (2017).

28. H. Mäntysaari and B. Schenke, Confronting impact parameter dependent JIMWLK evolution with HERA data, Phys. Rev. D98, p. 034013 (2018).

29. D. Mustaki, S. Pinsky, J. Shigemitsu and K. Wilson, Perturbative renormalization of null plane QED, Phys. Rev. D43, 3411 (1991).

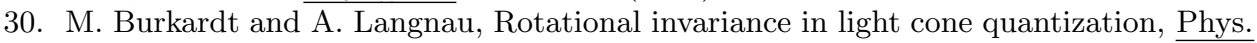
Rev. D44, 3857 (1991)

31. W.-M. Zhang and A. Harindranath, Light front QCD. 2: Two component theory, Phys. Rev. D48, 4881 (1993).

32. G. A. Chirilli, B.-W. Xiao and F. Yuan, Inclusive Hadron Productions in pA Collisions, Phys. Rev. D86, p. 054005 (2012).

33. A. Dumitru, A. Hayashigaki and J. Jalilian-Marian, The Color glass condensate and hadron production in the forward region, Nucl. Phys. A765, 464 (2006).

34. A. M. Stasto, B.-W. Xiao and D. Zaslavsky, Towards the Test of Saturation Physics Beyond Leading Logarithm, Phys. Rev. Lett. 112, p. 012302 (2014).

35. A. M. Stasto and D. Zaslavsky, Saturation in inclusive production beyond leading logarithm accuracy, Int. J. Mod. Phys. A31, p. 1630039 (2016).

36. E. Iancu, A. H. Mueller and D. N. Triantafyllopoulos, CGC factorization for forward particle production in proton-nucleus collisions at next-to-leading order, JHEP 12, p. 041 (2016). 
37. B. Ducloué, T. Lappi and Y. Zhu, Implementation of NLO high energy factorization in single inclusive forward hadron production, Phys. Rev. D95, p. 114007 (2017).

38. B. Ducloué, E. Iancu, T. Lappi, A. H. Mueller, G. Soyez, D. N. Triantafyllopoulos and Y. Zhu, Use of a running coupling in the NLO calculation of forward hadron production, Phys. Rev. D97, p. 054020 (2018).

39. G. A. Chirilli, B.-W. Xiao and F. Yuan, One-loop Factorization for Inclusive Hadron Production in $p A$ Collisions in the Saturation Formalism, Phys. Rev. Lett. 108, p. 122301 (2012).

40. B. Ducloué, T. Lappi and Y. Zhu, Single inclusive forward hadron production at next-to-leading order, Phys. Rev. D93, p. 114016 (2016). 